\title{
Conhecimento de mulheres da região metropolitana de Minas Gerais sobre o câncer de mama e sua prevenção
}

\author{
Knowledge of women from metropolitan region of Minas Gerais about breast cancer and \\ its prevention
}

\section{El conocimiento de las mujeres de la región metropolitana de Minas Gerais sobre el cáncer de mama y su prevención}

Luana Corrêa Cunha1, Flávio Santos Vasconcelos Barros ${ }^{1}$, Paula Vidigal Assumpção', Sarah Veloso Araújo Gomes ${ }^{1 *}$, Thomás Santiago Lopes Furtado ${ }^{1}$, Victória Mara Vieira Rocha ${ }^{1}$.

\section{RESUMO}

Objetivo: Conhecer as lacunas no entendimento do câncer de mama pelas mulheres de Minas Gerais. Métodos: Tratase de estudo observacional transversal de cunho quanti-qualitativo onde foram aplicados questionários em mulheres da região metropolitana de Minas Gerais e através da ferramenta online Google Forms. Questionário composto de três partes: (1) identificação da paciente; (2) história ginecológica-obstétrica; (3) prevenção do câncer de mama. A associação entre variáveis categóricas foi avaliada pelo teste Exato de Fisher. As análises foram realizadas no software $\mathrm{R}$ versão $4.0 .3 \mathrm{e}$ foi considerado nível de significância de 5\%. Resultados: 0 estudo incluiu 78 mulheres, sendo metade delas recrutadas no Ambulatório e outra metade via google forms. Dentre elas, 14,1\% fazem acompanhamento ginecológico a cada 2 anos ou mais frequentemente, $12 \%$ das mulheres julgaram não ser necessário realizar a mamografia quando na palpação das mamas não são encontradas alterações e 34\% não sabem como prevenir o câncer de mama. Conclusão: Muitas mulheres da região citada não sabem como prevenir o câncer de mama. Portanto, os profissionais de saúde capacitados devem orientar elas sobre a importância do acompanhamento com ginecologista, da realização de mamografia e de evitar os fatores de riscos modificáveis para o câncer de mama.

Palavras-chave: Mama, Doenças mamárias, Mamografia, Câncer de mama.

\begin{abstract}
Objective: Assessing gaps in the understanding of breast cancer and its prevention amongst women from metropolitan region of Minas Gerais, Brazil. Methods: This is a quanti-qualitative cross-sectional observational study in which questionnaires were applied to women from Minas Gerais through google forms. The questionnaire consists of three parts: (1) patient identification; (2) gynecological-obstetric history; (3) prevention of breast cancer. The association between categorical variables was assessed using Fisher's exact test. Analyzes were performed using the R software version 4.0 .3 and a $5 \%$ significance level was adopted. Results: 78 women were included, half of them recruited in the health service and the other half through google forms. Among them, $14.1 \%$ are followed up by a gynecologist every 2 years or more often, $12 \%$ of women reckoned it was not necessary to have a mammogram when there was no findings during breast examination, and 34\% do not know how to prevent breast cancer. Conclusion: Many women do not know how to prevent breast cancer. Therefore, trained health professionals should guide them about the importance of annual appointments with a gynecologist, having a mammogram and avoiding modifiable risk factors for breast cancer.
\end{abstract}

Keywords: Breast, Breast diseases, Mammography, Breast neoplasms.

\section{RESUMEN}

Objetivo: Conocer las brechas en la comprensión del cáncer de mama por parte de mujeres de la región metropolitana de Minas Gerais. Métodos: Estudio observacional transversal cuanti-cualitativo en que se aplicaron cuestionarios a mujeres de Minas Gerais mediante formularios de google. Cuestionario de tres partes: (1) identificación; (2) historial ginecológico-obstétrico; (3) prevención del cáncer de mama. La asociación entre variables categóricas se evaluó mediante la prueba exacta de Fisher. Los análisis se realizaron con el software $R$ versión 4.0 .3 y se consideró un nivel de significancia del $5 \%$. Resultados: Estudio incluyó a 78 mujeres, la mitad de ellas mujeres que se encontraban en la Clínica Ambulatoria y la otra mitad a través de formularios de google. 14,1\% con seguimiento ginecológico cada 2 años o más; $12 \%$ de las mujeres pensó que no era necesario hacerse una mamografía con la palpación de las mamas sin câmbios; $34 \%$ no sabe cómo prevenir el cáncer de mama. Conclusión: Mayoría de las mujeres no sabe cómo prevenir el cáncer. Por lo tanto, profesionales de la salud capacitados deben orientarlos sobre la importancia de realizar un seguimiento ginecológico, hacerse una mamografía y evitar factores de riesgo modificables para el cáncer.

Palabras clave: Mama, Enfermedades de la mama, Mamografía, Cáncer de mama.

${ }^{1}$ Faculdade Ciências Médicas de Minas Gerais (FCMMG), Belo Horizonte - MG. *E-mail: velosoagsarah@gmail.com 


\section{INTRODUÇÃO}

Segundo o Instituto Nacional do Câncer (INCA), o câncer (CA) de mama, ou carcinoma mamário, é uma doença heterogênea, com diversos fatores de risco, incluindo fatores ambientais e comportamentais: a idade avançada; a hereditariedade (BRCA1 e BRCA2); fatores endócrinos e história reprodutiva que ocasionem na elevação dos níveis de estrogênio INCA (2021). O subtipo mais comum é o ductal invasivo que, caso não seja feito o diagnóstico precoce, pode se metastatizar pelo corpo todo, aumentando a morbimortalidade da paciente. No artigo de Kameo SV, et al (2021) é ressaltado que, apesar do avanço no rastreamento e tratamento dessa neoplasia, a incidência está aumentando no Brasil (INCA, 2021)

De acordo com a Sociedade Brasileira de Mastologia, o CA de mama pode ser prevenido através de mudanças de hábitos de vida, principalmente para combater o sobrepeso e obesidade, associando ao rastreamento da neoplasia através da mamografia Chlebowski RT (2021). Além disso, na revisão integrativa realizada por Soares JCN, et al (2019) concluiu-se que a cada 12 meses de amamentação materna houve redução de 4\% no risco de desenvolver câncer de mama (SOARES ET AL., 2019; CHLEBOWSKI RT, 2021)

O câncer de mama é a segunda neoplasia mais comum em mulheres do mundo e do Brasil, sendo responsável por 30\% de todos os novos casos de câncer diagnosticados nesse gênero. Dados de 2018 do Instituto Nacional do Câncer, estimam 59.700 novos casos em 2018 e 2019, com um risco estimado de 56,33 casos a cada 100 mil mulheres no país. O risco cumulativo de uma brasileira desenvolver esse câncer aos 74 anos é de 6,33\% (INCA, 2021).

Em países como o Brasil, a detecção precoce dessa neoplasia ainda é um desafio, sendo a maioria dos diagnósticos realizados em estágios mais avançados da doença. Isso aumenta a morbidade e reduz a sobrevida das pacientes. Na tentativa de modificar esse contexto, o Ministério da Saúde tem incentivado a execução do diagnóstico precoce e do rastreamento (INCA, 2021; SILVA IS, 2018).

O diagnóstico precoce baseia-se na identificação dos primeiros sinais e sintomas da doença, buscando oferecer medidas assistenciais de qualidade em todas as etapas do acompanhamento das pacientes. Para que isso ocorra, é preciso que a população esteja alerta para as características da neoplasia, os profissionais de saúde capacitados para avaliação dos casos suspeitos e os serviços de saúde preparados para confirmação dos casos (MIGOWSKI A, et al., 2018; INCA, 2021).

O rastreamento do câncer de mama é feito por meio de exames em mulheres hígidas na tentativa de identificar a doença em sua fase assintomática. Atualmente, o único teste de rastreamento preconizado pelo Ministério da Saúde para identificação precoce do câncer de mama é a mamografia. No entanto, este exame só deve ser realizado bienalmente por mulheres entre 50 e 69 anos (INCA, 2021). Dados da literatura demonstram que no Brasil somente $35 \%$ das mulheres entre 50 e 69 anos realizam rastreamento de câncer de mama apropriado. Além disso, $45 \%$ das mamografias realizadas no país são feitas em mulheres com menos de 50 anos Hallowell BD, et al. (2017). A educação e o conhecimento da população sobre essa neoplasia são imprescindíveis para o seu controle. Em função disso, diversas pesquisas buscaram avaliar o entendimento de mulheres acerca do câncer de mama (CHAKA B, et al., 2018; GONÇALVES CV, et al., 2018; OLIVEIRA DA, et al., 2020).

Um estudo conduzido por Chaka et al. (2018), verificou que os principais fatores desfavoráveis ao rastreamento adequado do câncer de mama foram a falta de conhecimento sobre a existência da doença e a falta de educação em geral das participantes. Em soma a esses estudos, Gonçalves CV (2017) publicou um artigo em 2018, em que mulheres com menor nível de instrução, classes econômicas mais baixas e aquelas de outra etnia que não a branca, apresentaram menor esclarecimento quanto aos exames clínicos e imaginológicos de prevenção ao CA de mama, o que reduz a autonomia em saber como cuidar da própria saúde. Além disso, segundo o estudo feito por Oliveira DA (2020), existe um déficit no conhecimento acerca dos fatores de risco para o câncer de mama, até mesmo para acadêmicos de diversos cursos de ciência da saúde (CHAKA B, et al., 2018; GONÇALVES CV, et al., 2018; OLIVEIRA DA, et al., 2020).

Por isso, conhecer as lacunas no entendimento do câncer de mama pela população é fundamental para o desenvolvimento de estratégias de intervenção individualizadas e eficazes no combate à morbimortalidade 
dessa neoplasia. Desta forma, o presente estudo possui o objetivo de investigar o conhecimento das mulheres acerca dessa patologia, relacionando os dados encontrados aos fatores de risco e nível de escolaridade.

\section{MÉTODOS}

Trata-se de um estudo observacional transversal de cunho quanti-qualitativo que prevê a avaliação do nível de conscientização da população feminina acerca do câncer de mama e seu rastreamento. A população estudada foi de mulheres por meio de entrevista, sendo que metade delas foram abordadas em via pública na região central do Estado de Minas Gerais, e a outra metade através da ferramenta online para questionários Google Forms.

O questionário aplicado é composto de três partes, sendo a primeira composta pela identificação do paciente (idade, sexo, estado civil, escolaridade, renda média mensal, tabagismo, consumo de bebidas alcóolicas), a segunda sobre a história ginecológica-obstétrica (idade da menarca, ciclos menstruais, uso de contraceptivos hormonais, número de gestações, idade da primeira gestação, amamentação, idade da menopausa, terapia de reposição hormonal e acompanhamento ginecológico) e a terceira abordando a investigação sobre a prevenção do câncer de mama (se sabe o que é mamografia; se já fez alguma mamografia e há quanto tempo realizou a última; se sabe o que é o autoconhecimento das mamas; se tem o hábito de palpar as mamas; se julga necessário realizar mamografia, ainda que a palpação das mamas não indiquem alteração; qual o maior risco para desenvolvimento do câncer de mama; o que a levaria a buscar atendimento médico para verificar presença de câncer de mama; se sabe quais são as medidas preventivas mais importantes para o câncer de mama).

Foi realizada pesquisa nas bases de dados Lilacs (Latin American and Caribbean Health Sciences Literature), PubMed, Medline (Medical Literature Analysis and Retrieval System Online), Scielo (Scientific Electronic Library Online) e Cochrane Library e em textos publicados pelo Ministério da Saúde e pelo INCA. Foram selecionados artigos em português e inglês publicados desde o ano 2016 que apresentaram relevância para a pesquisa após análise do resumo. Em seguida, os estudos foram avaliados quanto à qualidade da evidência, de acordo com o critério GRADE (Grading of Recommendatons Assessment, Development and Evaluaton), que visa à criação de um sistema universal, transparente e sensível para graduar a qualidade das evidências e a força das recomendações.

O cálculo amostral foi realizado considerando um nível de significância de $5 \%$ e um poder de $80 \%$, sendo o tamanho amostral de 39 mulheres por grupo, totalizando 78 indivíduos pesquisados. Foram incluídas no projeto mulheres com idade igual ou superior a 18 anos, de qualquer raça, tipo físico, orientação sexual e qualquer classe social. Os critérios de exclusão, por sua vez, serão homens com identidade de gênero feminino e mulheres: analfabetas; incapazes de responder o questionário por debilitação física ou mental; já diagnosticadas com câncer de mama.

As variáveis numéricas foram apresentadas como média \pm desvio-padrão e mediana ( 1 - quartil -3 quartil) e as variáveis categóricas, como frequências absolutas e relativas. A associação entre variáveis categóricas foi avaliada via teste Exato de Fisher. As análises foram realizadas no software $R$ versão 4.0 .3 e foi considerado nível de significância de $5 \%$.

O estudo protegeu os participantes da pesquisa científica envolvendo seres humanos e considerou o respeito à dignidade humana como primordial e necessário para a pesquisa. Além disso, assegurou manter em sigilo as informações que os participantes forneceram. O projeto foi submetido ao Comitê de Ética em Pesquisa com o Certificado de Apresentação de Apreciação Ética (CAAE) número 04495218.3.0000.5134 e de acordo com os artigos 13 e 11, Resolução CNS no 446/2011; item X.1, 3, a, Resolução CNS ํㅡ 466/2012 e item 2.1 da Norma Operacional CNS n 001/2013.

\section{RESULTADOS}

A partir do questionário, foram analisadas 78 mulheres com idade média de $47,0 \pm 14,4$ anos. A população pesquisada é representada na Tabela 1. Especificamente de 20-49 anos: 39, 50-69 anos: 33 e acima de 70 anos: 5 . 
Tabela 1 - Caracterização das mulheres e história ginecológica-obstétrica das mulheres pesquisadas quanto ao conhecimento do câncer de mama, $\mathrm{n}=78$.

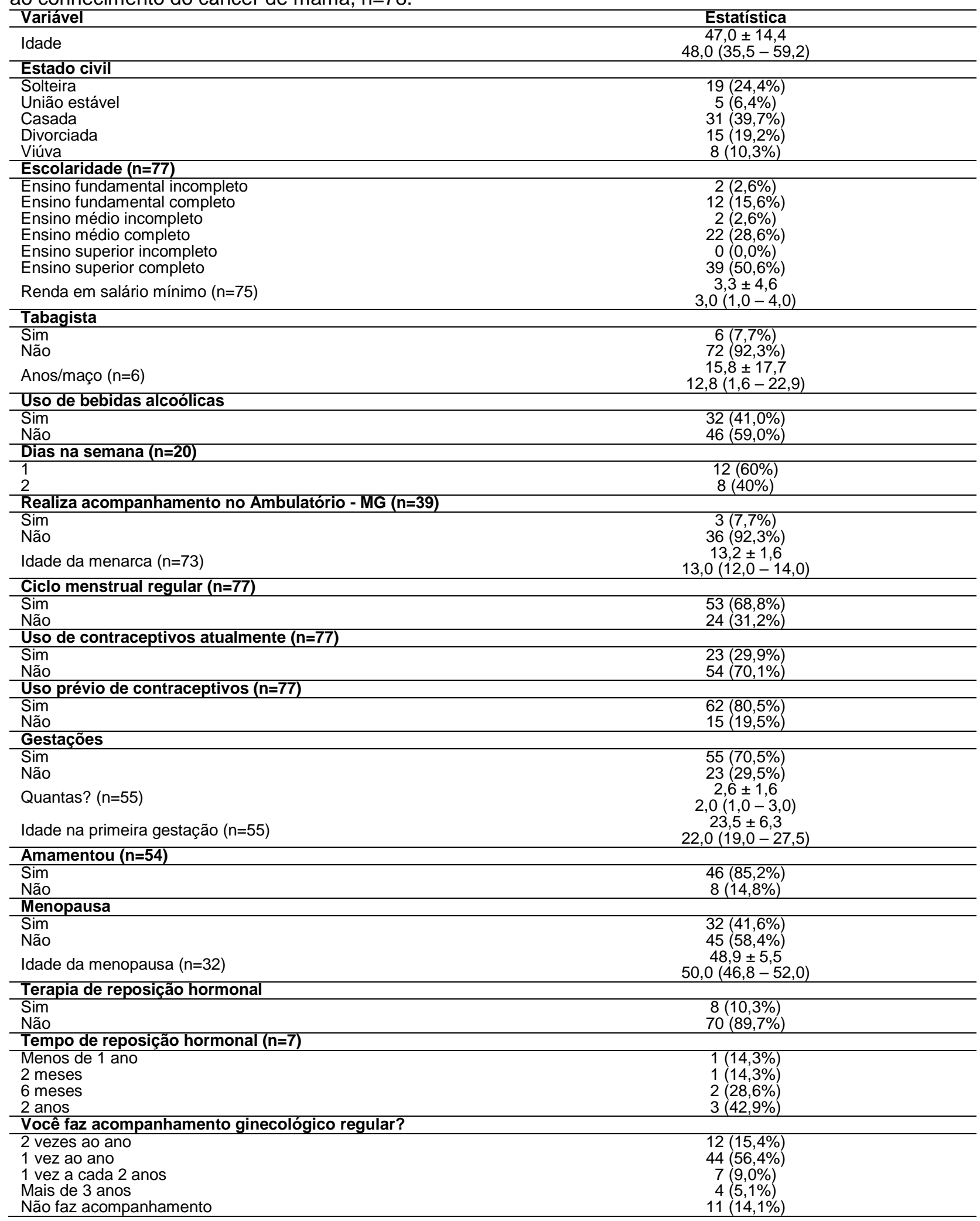

Fonte: Cunha LC, et al., 2021.

Com relação ao conhecimento dessas mulheres sobre o câncer de mama, foi obtido dados relacionados ao acompanhamento médico mamográfico das mulheres entrevistadas, traduzidos na Tabela 2. Nesse sentido, foram avaliados o conhecimento sobre mamografia e últimos exames realizados. Outro aspecto 
avaliado foi em relação aos cuidados com a mama, tanto em relação ao autoconhecimento quanto à percepção de possíveis alterações e até fatores de risco. Associados ao câncer de mama.

Tabela 2 - Investigação do conhecimento da prevenção do câncer de mama pelas mulheres pesquisadas $(n=78)$.

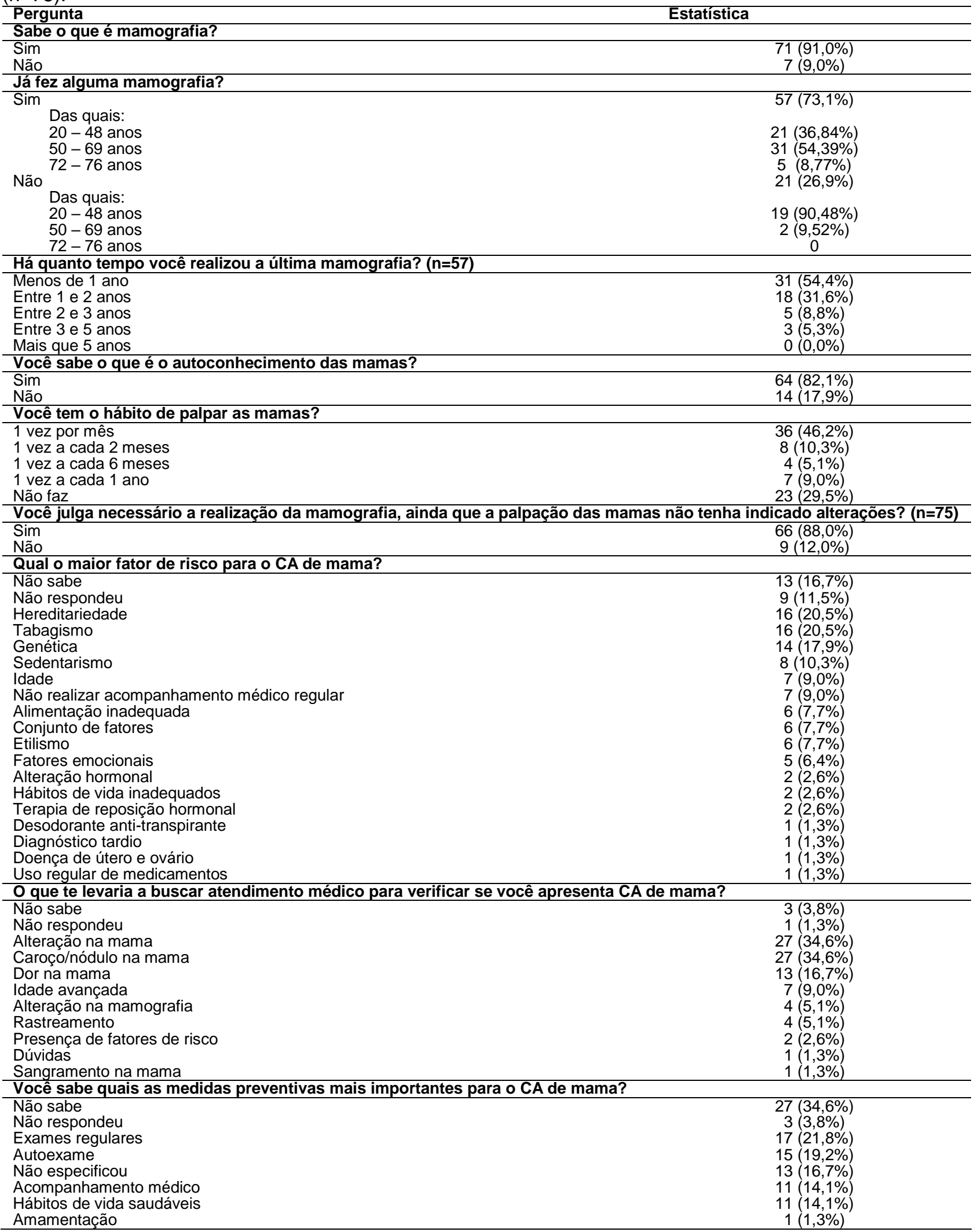

Fonte: Cunha LC, et al., 2021. 
Considerando o público heterogêneo quanto à escolaridade, foi pesquisado a importância deste dado para o conhecimento acerca do rastreamento do câncer de mama. Na Tabela 3 foram relacionadas as variáveis de escolaridade ao nível de conhecimento mamográfico das mulheres entrevistadas. Não foi observada diferença estatística significativa entre os grupos, ou seja, o conhecimento das mulheres sobre o câncer de mama não variou com a escolaridade.

Tabela 3 - Comparação da escolaridade e conhecimento da prevenção do câncer de mama pelas mulheres pesquisadas, $n=78$.

\begin{tabular}{|c|c|c|c|c|c|}
\hline \multirow[b]{2}{*}{ Pergunta } & \multicolumn{4}{|c|}{ Escolaridade } & \multirow[b]{2}{*}{ Valor-p $\mathrm{p}^{\mathrm{F}}$} \\
\hline & $\begin{array}{l}\text { Fundamental } \\
\text { incompleto }\end{array}$ & $\begin{array}{l}\text { Fundamental } \\
\text { completo }\end{array}$ & $\begin{array}{l}\text { Médio } \\
\text { completo }\end{array}$ & $\begin{array}{l}\text { Superior } \\
\text { completo }\end{array}$ & \\
\hline Sabe o que é mamografia? & & & & & 0,315 \\
\hline Sim & $2(100,0)$ & $11(78,6)$ & $20(90,9)$ & $37(94,9)$ & \\
\hline Não & $0(0,0)$ & $3(21,4)$ & $2(9,1)$ & $2(5,1)$ & \\
\hline \multicolumn{5}{|c|}{ Você sabe o que é o autoconhecimento das mamas? } & $<0,001$ \\
\hline Sim & $1(50,0)$ & $6(42,9)$ & $19(86,4)$ & $37(94,9)$ & \\
\hline Não & $1(50,0)$ & $8(57,1)$ & $3(13,6)$ & $2(5,1)$ & \\
\hline \multicolumn{5}{|c|}{$\begin{array}{l}\text { Você julga necessário a realização da mamografia, ainda que a palpação das mamas } \\
\text { não tenha indicado alterações? }(n=75)\end{array}$} & 0,409 \\
\hline Sim & $1(50,0)$ & $11(91,7)$ & $19(90,5)$ & $34(87,2)$ & \\
\hline Não & $1(50,0)$ & $1(8,3)$ & $2(9,5)$ & $5(12,8)$ & \\
\hline
\end{tabular}

Legenda: Teste Exato de Fisher.

Fonte: Cunha LC, et al., 2021.

$\mathrm{Na}$ Tabela 4, foram recrutados dados sobre as alterações na mama que levariam a entrevista a buscar atendimento médico, seja caroço, nódulo, dor, entre outras alterações. Esses dados foram relacionados, posteriormente, ao nível de escolaridade das entrevistadas.

Tabela 4 - Avaliação da escolaridade para as respostas da questão "O que te levaria a buscar atendimento médico para verificar se você apresenta CA de mama?", n=78.

\begin{tabular}{lccccc}
\hline \multirow{2}{*}{ Escolaridade } & \multicolumn{2}{c}{ O que te levaria a buscar atendimento médico para verificar se } \\
& \multicolumn{5}{c}{ você apresenta CA de mama? } \\
\cline { 2 - 6 } & $\begin{array}{c}\text { Alteração } \\
\text { na mama }\end{array}$ & $\begin{array}{c}\text { Caroço/nódul } \\
\text { o na mama }\end{array}$ & $\begin{array}{c}\text { Dor na } \\
\text { mama }\end{array}$ & $\begin{array}{c}\text { Sangramento } \\
\text { na mama }\end{array}$ & $\begin{array}{c}\text { Não } \\
\text { sabe }\end{array}$ \\
\hline Fundamental incompleto & $0(0,0)$ & $1(3,7)$ & $0(0,0)$ & $0(0,0)$ & $0(0,0)$ \\
Fundamental completo & $1(3,7)$ & $5(18,5)$ & $4(33,3)$ & $1(100,0)$ & $2(66,7)$ \\
Médio incompleto & $0(0,0)$ & $1(3,7)$ & $0(0,0)$ & $0(0,0)$ & $0(0,0)$ \\
Médio completo & $6(22,2)$ & $11(40,7)$ & $3(25,0)$ & $0(0,0)$ & $1(33,3)$ \\
Superior incompleto & $0(0,0)$ & $0(0,0)$ & $0(0,0)$ & $0(0,0)$ & $0(0,0)$ \\
Superior completo & $20(74,1)$ & $9(33,3)$ & $5(41,7)$ & $0(0,0)$ & $0(0,0)$ \\
\hline
\end{tabular}

Fonte: Cunha LC, et al., 2021.

\section{DISCUSSÃO}

No presente estudo, observou-se que, do total de mulheres entrevistadas, 33 (42,3\%) encontravam-se dentro da faixa etária preconizada para rastreamento do câncer de mama de acordo com o Ministério da Saúde. Dessas 33 pacientes, 2 (6\%) nunca realizaram a mamografia. Esse dado é relevante tendo em vista que a mamografia é um exame acessível tanto para a população assistida pelo Sistema Único de Saúde quanto pelo sistema privado, e representa o principal método rastreio e diagnóstico precoce do câncer de mama.

Pôde-se observar, também, que $71(91 \%)$ mulheres entrevistadas sabiam dizer o que é a mamografia. No entanto, 27 (34,6\%) não souberam especificar quais são as medidas preventivas para o câncer de mama e $15(19,2 \%)$ responderam "autoexame" como medida preventiva. Esses dados corroboram para a suspeita de que há um certo conhecimento generalizado sobre câncer de mama entre as entrevistadas, entretanto, aspectos mais específicos e igualmente importantes são pouco abordados e disseminados. 
Uma hipótese que foi avaliada neste trabalho é se há plena compreensão da diferença entre os termos "autoconhecimento" e "autoexame". É possível que muitas das mulheres entrevistas tenham respondido saber sobre o auto-conhecimento da mama, mas na verdade se referiam ao auto-exame. Sendo assim, é fundamental informar a todas as mulheres sobre a diferença.

Recentemente, muitas autoridades médicas têm promovido a política de alerta à saúde das mamas. Esta medida incentiva a prática da palpação das mamas, sempre que a mulher se sinta confortável, com o objetivo de conhecer a forma e a textura desse órgão. Ao identificar alterações, como dor, secreção mamária e massas, ela deve procurar assistência para avaliação segundo o American College of Obstetricians and Gynecologists (ACOG) (2017).

Esta estratégia se diferencia do autoexame das mamas, pois não preconiza uma avaliação sistemática e rotineira das mamas. Com isso, tem se mostrado mais eficaz na identificação de lesões neoplásicas (INCA, 2021). Entretanto, o autoexame, como prática isolada para rastreamento para câncer de mama, deixou de ser uma prática recomendada pelo Ministério da Saúde, devendo ser realizado em associação a exames de imagem regulares e acompanhamento médico. Esses dados demonstram pouco conhecimento da população alvo acerca das medidas preventivas eficientes e importantes para a prevenção da doença e até mesmo quando é necessário buscar atendimento médico (Tabela 3). Sendo assim, esses resultados são considerados alarmantes no que tange a informação e conhecimento sobre o câncer de mama.

No que se refere às respostas obtidas com o nível de escolaridade das entrevistadas, percebeu-se que informações gerais, como "o que é mamografia", são de conhecimento da maioria (91\%), independentemente do nível de escolaridade. Entretanto, ao relacionar a escolaridade com o conhecimento mais aprofundado sobre o câncer de mama, através das perguntas: "qual o maior fator de risco para o câncer de mama?", "o que te levaria a buscar atendimento médico para verificar se você tem câncer de mama?" ou mesmo "você sabe quais as medidas preventivas mais importantes para o câncer de mama?", as respostas também não são correspondentes ao nível de escolaridade. Isso reforça a necessidade de promover a disseminação de informações corretas acerca de conhecimentos gerais, prevenção e conduta sobre o câncer de mama para a população de mulheres da região metropolitana de Minas Gerais, independente do grau de instrução.

Dentre as que apresentam escolaridade de, no mínimo, ensino médio completo, 4 (6,56\%) mulheres não souberam dizer o que é mamografia. Além disso, dentre essas mulheres com no mínimo ensino médio completo, 55 afirmaram que só procurariam atendimento médico para verificar se há risco de câncer de mama, após verificar alguma alteração no órgão. Esta informação é convergente com o que amplamente se obtém na literatura, tendo em vista que, mesmo com o avanço da medicina preventiva por meio dos métodos de rastreamento para o câncer de mama, muitas mulheres ainda descobrem essa patologia em estágio avançado. Um estudo publicado por Traldi MC, et al. (2016), demonstra uma demora média maior que 2,4 vezes o tempo máximo de espera de 60 dias para o diagnóstico (TRALDI MC, et al. 2016)

De acordo com a medicina preventiva, é possível atuar precocemente no período de patogênese, ou seja, antes da doença se instalar, tanto através da prevenção primária quanto secundária. No que se refere à prevenção primária, cabe destacar que a identificação de fatores de risco para o câncer de mama é um dos principais pilares na prevenção dessa patologia. Diante disso, é fundamental que a população conheça seus fatores de risco para uma atuação sobre eles. $\mathrm{Na}$ análise deste estudo, observou-se que $28,2 \%$ das entrevistadas não souberam citar nenhum fator de risco para câncer de mama ou não responderam, o que é um resultado insatisfatório frente à relevância do assunto e à facilidade de se informar sobre tais fatores, principalmente aqueles de risco modificáveis, como a obesidade e tabagismo (BATISTA GV, et al., 2020).

Dentre as respostas obtidas referentes aos fatores de risco, a grande maioria citou fatores modificáveis, como sedentarismo, hábitos de vida inadequados (tabagismo e/ou etilismo) e alimentação inadequada. Esses dados corroboram a importância da assistência multiprofissional, uma vez que são elementos que podem ser controlados diante de recomendação e acompanhamento adequados. Em linhas gerais, das 78 mulheres, apenas $4(5,13 \%)$ citam o rastreamento como método para descoberta do câncer de mama; aqui, caberia a atuação da prevenção secundária que preconiza diagnósticos precoces e em fases subclínicas e, 
consequentemente, tratamento precoces, visando-se a melhoria do prognóstico. Essas duas modalidades são as mais cruciais na redução de danos e, por isso, destacamos a importância da informação como a principal forma de evitar o câncer de mama e suas repercussões.

As limitações do trabalho relacionam-se ao formulário online que possibilitou respostas discursivas, o que aumentou a imprecisão das mesmas: seja por ausência de resposta ou discrepância na escrita. Além disso, por depender da confiabilidade nas respostas, há o risco de viés de memória.

\section{CONCLUSÃO}

Uma amostra das mulheres da região metropolitana de Minas Gerais não sabe como prevenir o câncer de mama, e observou-se que isto independe do nível de escolaridade das mesmas. Portanto, é de suma importância que essas mulheres sejam informadas e orientadas pelos profissionais de saúde capacitados sobre os benefícios do acompanhamento regular com o ginecologista, realização da mamografia e sobre a atuação nos fatores de riscos modificáveis para o câncer de mama. É importante ressaltar que cabe às autoridades públicas de saúde implementar ações de conscientização sobre o câncer de mama a fim de suplantar a lacuna de conhecimento dessa patologia.

\section{REFERÊNCIAS}

1. AMERICAN COLLEGE OF OBSTETRICIANS AND GYNECOLOGISTS (ACOG). Breast Cancer Risk Assessment and Screening in Average-Risk Women. The American College of Obstetricians and Gynecologists, $2017 ; 179$.

2. BATISTA GV, et al. Câncer de mama: fatores de risco e métodos de prevenção. Research, Society and Development, 2020; 9(12).

3. CHAKA B, et al. A survey of knowledge and attitudes relating to cervical and breast cancer among women in Ethiopia. BMC Public Health, 2018; 18(1072).

4. CHAVAGLIA SRR, et al. Public actions for control of breast cancer in Brazil: integrative review. Revista Brasileira Enfermagem. 2016; 69(4): 746-55

5. CHLEBOWSKI RT. Fatores que modificam o risco de câncer de mama em mulheres. UptoDate, 2021; Disponível em: UptoDate. Acessado em: 2021.

6. CORREA CS, et al. Breast Cancer screening in Minas Gerais: assessment of data from information health systems of the Brazilian National Health System. Epidemiologia e Serviços em Saúde, 2017; 26(3): 481-492.

7. GONÇALVES CV, et al. O conhecimento de mulheres sobre os métodos para prevenção secundária do câncer de mama. Ciência \& Saúde Coletiva, 2017; 22: 4073-4082.

8. INSTITUTO NACIONAL DE CÂNCER JOSÉ ALENCAR GOMES DA SILVA. Detecção precoce do câncer / Instituto Nacional de Câncer José Alencar Gomes da Silva. - Rio de Janeiro: INCA, 2021. Disponível em: https://www.inca.gov.br/sites/ufu.sti.inca.local/files//media/document//deteccao-precoce-do-cancer.pdf. Acessado em: Agosto/2021.

9. INSTITUTO NACIONAL DE CÂNCER JOSÉ ALENCAR GOMES DA SILVA. Parâmetros técnicos para rastreamento do câncer de mama. / Instituto Nacional de Câncer José Alencar Gomes da Silva. - Rio de Janeiro: INCA, 2021. ISBN 978-65-88517-25-3. Disponível em: https://www.inca.gov.br/sites/ufu.sti.inca.local/files//media/document/parametrostecrastreamentocamama_2021_1.pd f. Acessado em Agosto/2021.

10. HALLOWELL BD, et al. Breast cancer related perceptions and practices of health professionals working in Brazil's network of primary care units. Preventive Medicine, 2018; 106: 216-223.

11. KAMEO SV, et al. Clinical-epidemiological profile of women undergoing oncological treatment for invasive ductal breast carcinoma. Research, Society and Development, [S. I.], v. 10, n. 1, p. e39110111836, 2021. DOI: 10.33448/rsdv10i1.11836. Disponível em: https://rsdjournal.org/index.php/rsd/article/view/11836. Acesso em: setembro/2021

12. KLUG J, et al. Screening for breast and cervical cancer in a large German city: participation, motivation and knowledge of risk factors. European Journal of Public Health, 2005; 15 (1): 70-77.

13. MIGOWSKI A, et al. Diretrizes para detecção precoce do câncer de mama no Brasil. II-Novas recomendações nacionais, principais evidências e controvérsias. Cadernos de Saúde Pública, 2018; 34(6).

14. OLIVEIRA DA, et al. Autocuidado e prevenção do câncer de mama: conhecimento das estudantes de graduação em saúde. Revista Eletrônica Acervo Saúde, 2020; 12(10): e4429-e4429.

15. SILVA IS. Políticas de controle do câncer de mama no Brasil: quais são os próximos passos? Cadernos de Saúde Pública, 2018; 34(6).

16. SILVA N, et al. Conhecimento de mulheres sobre o câncer de mama e de colo do útero. Paidéia, 2018; 15(32): 409416.

17. SOARES JCN, et al. Aleitamento materno na prevenção do câncer de mama: uma revisão integrativa da literatura. Revista Uningá, 2019; 56(S6): 13-22.

18. TRALDI, MC, et al. Delay in breast cancer diagnosis on women assisted in the Public Health System. Caderno de Saúde Coletiva, 2016; 24(2): 185-191. 